\title{
Gain in stochastic resonance: Precise numerics versus linear response theory beyond the two-mode approximation
}

\author{
Jesús Casado-Pascual, Claus Denk, José Gómez-Ordóñez, and Manuel Morillo \\ Física Teórica, Universidad de Sevilla, Apartado de Correos 1065, Sevilla 41080, Spain \\ Peter Hänggi \\ Institut für Physik, Universität Augsburg, Universitätsstraße 1, D-86135 Augsburg, Germany
}

(Received 26 November 2002; published 18 March 2003)

\begin{abstract}
In the context of the phenomenon of stochastic resonance (SR), we study the correlation function, the signal-to-noise ratio (SNR), and the ratio of output over input SNR, i.e., the gain, which is associated to the nonlinear response of a bistable system driven by time-periodic forces and white Gaussian noise. These quantifiers for SR are evaluated using the techniques of linear response theory (LRT) beyond the usually employed two-mode approximation scheme. We analytically demonstrate within such an extended LRT description that the gain can indeed not exceed unity. We implement an efficient algorithm, based on work by Greenside and Helfand (detailed in the Appendix), to integrate the driven Langevin equation over a wide range of parameter values. The predictions of LRT are carefully tested against the results obtained from numerical solutions of the corresponding Langevin equation over a wide range of parameter values. We further present an accurate procedure to evaluate the distinct contributions of the coherent and incoherent parts of the correlation function to the SNR and the gain. As a main result we show for subthreshold driving that both the correlation function and the SNR can deviate substantially from the predictions of LRT and yet the gain can be either larger or smaller than unity. In particular, we find that the gain can exceed unity in the strongly nonlinear regime which is characterized by weak noise and very slow multifrequency subthreshold input signals with a small duty cycle. This latter result is in agreement with recent analog simulation results by Gingl et al. [ICNF 2001, edited by G. Bosman (World Scientific, Singapore, 2002), pp. 545-548; Fluct. Noise Lett. 1, L181 (2001)].
\end{abstract}

DOI: 10.1103/PhysRevE.67.036109

PACS number(s): 05.10.Gg, 05.40.-a, 02.50.-r

\section{INTRODUCTION}

Over the past 20 years or so, a large amount of work has been devoted to the study of the dynamics of noisy nonlinear systems driven by external periodic forces. One of the main reasons for this interest is related to the phenomenon of stochastic resonance (SR) [1-5], namely, the possibility of using the concerted action of noise and nonlinearity to augment selectively, for some parameter values, the output of the nonlinear system with respect to what it would be for a linear system dynamics.

The two common quantifiers for stochastic resonance are the spectral amplification measure $[2,6,7]$ and the signal-tonoise ratio (SNR) $[2,8]$. They are defined in terms of the Fourier components of the correlation function associated to the stochastic variable, $x(t)$. Due to the periodicity of the driving force, the stochastic process $x(t)$ is explicitly nonstationary. Thus, the two-time function $\langle x(t+\tau) x(t)\rangle$ depends on both $t$ and $\tau$. For very large values of $t$, this quantity is periodic in $t$ with the period of the external driving. Thus, its cycle average over one period of $t$ yields a function of just $\tau$ : the correlation function, $C(\tau)$. The analysis of its structure reveals that $C(\tau)$ is the sum of two terms [2]: One term is periodic in $\tau$ with the same period as the driving force and it is called the coherent part, $C_{c o h}(\tau)$. The other term, the incoherent part $C_{\text {incoh }}(\tau)$, decays to zero for $\tau \rightarrow \infty$. The SNR of the output process $x(t)$, denoted by $R_{\text {out }}$, is defined as the ratio of the amplitude of the Fourier mode of the coherent part at the driving frequency, and the power spectral density of the incoherent part taken also at the driving frequency. By definition, $R_{\text {out }}$ is thus a dimensional quantity.

The SNR of an input signal, $R_{\text {inp }}$, containing the sum of the external driving and the Gaussian white noise, can easily be evaluated. A convenient dimensionless parameter, the gain $G$, defined by the ratio of $R_{\text {out }}$ over $R_{\text {inp }}$ can then be introduced. For the case that the Langevin dynamics is linear in $x$ driven by additive white Gaussian noise, the output SNR is exactly the same as that of the input; that is, the gain assumes precisely the value unity. In a general nonlinear case, neither the output SNR nor the gain can be evaluated exactly by analytical means. As a consequence, their evaluation necessarily requires approximate procedures.

It was pointed out previously that the gain of a noisy nonlinear dynamical system subject to subthreshold signals cannot exceed $1[9,10]$. This feature has been rationalized using the ideas of linear response theory (LRT), thought to be valid for weak driving amplitudes and not too small noise strengths. It should be pointed out, however, that the validity of LRT critically depends also on the value of the frequency, as has convincingly been demonstrated in recent works $[11,12]$

In the context of LRT theory it has been pointed out in Ref. [9] that a corollary of LRT is that "for small amplitude signals, the signal-to-noise ratio at the output of a system driven by a stationary Gaussian noise does not exceed that at the input, even if the system displays SR." Moreover, in Ref. [10], the authors state that for "small signal in a Gaussian noise background, it is a theorem that the SNR at the output 
of a nonlinear device must be less than or equal to the SNR at the input." On the other hand, studies on nondynamical systems [13-16], on dynamical systems driven by large amplitude sinusoidal forces [17], and on dynamical systems driven by pulsed (multifrequency) periodic forces with subthreshold amplitudes $[18,19]$, have reported gains larger than unity. Clearly, for this to occur, the stochastic system must operate in a regime where LRT does not apply. It is therefore of interest to delineate carefully the limit of applicability of the LRT description of the correlation function, the SNR, and the gain of a nonlinear noisy driven system.

In this paper, we have tackled this challenge by carrying out a detailed numerical evaluation of the correlation function $C(\tau)$ and its components, $C_{c o h}(\tau)$ and $C_{\text {incoh }}(\tau)$, of the SNR and the gain of a bistable noisy system which is driven by time-periodic forces. The numerical predictions have been compared with those provided by the LRT approximation that accounts for the full spectrum of all relaxation modes.

As it is well known, LRT requires the knowledge of the system susceptibility, or alternatively, of the correlation function of the noisy system in the absence of driving, $K(t)$ $[2,11,12,20-22]$. None of these quantities are known exactly for nonlinear systems. For sufficiently small values of the noise strength, suitable analytical approximations to $K(t)$ can been used $[2,11,12,20,21]$. On the other hand, for large values of the noise intensity, we have evaluated $K(t)$ from the numerical solution of the Fokker-Planck equation using an adaptation of the split operator technique of Feit et al. [23], as it has been detailed in Ref. [24]. In this paper, we also present a detailed proof of the statement that within LRT, the gain $G^{(L R T)} \leqslant 1$, by use of the full spectral approach; this proof differs from alternative attempts in Refs. $[9,10]$ which use additional restrictions such as a linear response theory for the fluctuations themselves.

The "typical" procedure to evaluate the SNR involves the Fourier analysis of a very long record of the stochastic trajectory, $x(t)$. Using the fast Fourier transform (FFT) of the record, the corresponding periodogram is constructed. There are several drawbacks with this procedure. There are subtleties inherent to the interpretation and evaluation of the periodogram (see for instance the critical comments in Ref. [25]). There are also major problems associated with the fact that the power spectrum contains $\delta$ peaks at the driving frequency and its higher harmonics arising from the coherent part of the correlation function. The contribution of the incoherent part at those frequencies is embedded in those peaks, and it is not a simple task to estimate the separate contribution to the peaks of the coherent and incoherent parts of the periodogram. The evaluation of the SNR gain requires a good knowledge of both contributions, and any small error in the estimation of the incoherent contribution yields unreasonable values for the gain. Indeed, in our opinion, a much better estimate would be obtained if the periodic part of the output signal were subtracted from the data before performing its FFT.

In this work, we propose such an alternative procedure. The Langevin equation is numerically integrated for a large number of noise realizations. The time evolution of the correlation function and its coherent part are directly evaluated from the numerical solution after averaging over the noise realizations. The incoherent part is obtained from the difference $C_{\text {incoh }}(\tau)=C(\tau)-C_{c o h}(\tau)$. As the definition of the SNR involves the amplitude of the Fourier mode of $C_{c o h}(\tau)$ and the spectral density of $C_{\text {incoh }}(\tau)$ just at the driving frequency, the evaluation of $R_{\text {out }}$ requires just two numerical quadratures; that is, there is no need to construct the full spectrum.

The paper is organized as follows. In the following section, we introduce the model and provide definitions of the quantities of interest. In Sec. III, the main points of the LRT description of the correlation functions are detailed. We also present in this section a different and straightforward proof of the fact that $G^{(L R T)} \leqslant 1$, based on the spectral properties of the Fokker-Planck operator, and its adjoint, in the absence of driving. In Sec. IV, we present the numerical procedure used to obtain the correlation function, the SNR, and the gain from the numerical solution of the Langevin equation. The very efficient algorithm used in this work is summarized in the Appendix. The numerical results are compared with the predictions of LRT for a variety of parameters and two distinct types of driving forces: a monochromatic force and a periodic sequence of pulses. Finally, we present conclusions for the main findings of our work.

\section{CORRELATION FUNCTION, SIGNAL-TO-NOISE RATIO, AND GAIN}

Let us consider a system characterized by a single degree of freedom, $x$, subject to the action of a zero average Gaussian white noise with $\langle\xi(t) \xi(s)\rangle=2 D \delta(t-s)$ and driven by an external periodic signal $F(t)$ with period $T$. In the Langevin description, its dynamics is generated by the equation

$$
\dot{x}(t)=-U^{\prime}[x(t)]+F(t)+\xi(t) .
$$

The corresponding linear Fokker-Planck equation (FPE) for the probability density $P(x, t)$ reads

$$
\frac{\partial}{\partial t} P(x, t)=\hat{\mathcal{L}}(t) P(x, t),
$$

where

$$
\hat{\mathcal{L}}(t)=\frac{\partial}{\partial x}\left[U^{\prime}(x)-F(t)+D \frac{\partial}{\partial x}\right] .
$$

In the expressions above, $U^{\prime}(x)$ represents the derivative of the potential $U(x)$. The periodicity of the external driving $F(t)$ allows its Fourier series expansion in the harmonics of the fundamental frequency $\Omega=2 \pi / T$, i.e.,

$$
F(t)=\sum_{n=1}^{\infty}\left[f_{n} \cos (n \Omega t)+g_{n} \sin (n \Omega t)\right],
$$

with the Fourier coefficients, $f_{n}$ and $g_{n}$, given by

$$
f_{n}=\frac{2}{T} \int_{0}^{T} d t F(t) \cos (n \Omega t),
$$




$$
g_{n}=\frac{2}{T} \int_{0}^{T} d t F(t) \sin (n \Omega t) .
$$

Here, we are assuming that the cycle average of the external driving over its period equals zero.

The two-time correlation function $\langle x(t+\tau) x(t)\rangle_{\infty}$ in the limit $t \rightarrow \infty$ is given by

$$
\begin{aligned}
\langle x(t+\tau) x(t)\rangle_{\infty}= & \int_{-\infty}^{\infty} d x^{\prime} x^{\prime} P_{\infty}\left(x^{\prime}, t\right) \\
& \times \int_{-\infty}^{\infty} d x x P_{1 \mid 1}\left(x, t+\tau \mid x^{\prime}, t\right),
\end{aligned}
$$

where $P_{\infty}(x, t)$ is the time-periodic, asymptotic long time solution of the FPE and the quantity $P_{1 \mid 1}\left(x, t+\tau \mid x^{\prime}, t\right)$ denotes the two-time conditional probability density that the stochastic variable will have a value near $x$ at time $t+\tau$ if its value at time $t$ was exactly $x^{\prime}$. It can been shown [2,7] that, in the limit $t \rightarrow \infty$, the two-time correlation function $\langle x(t$ $+\tau) x(t)\rangle_{\infty}$ becomes a periodic function of $t$ with the period of the external driving. Then, we define the one-time correlation function $C(\tau)$ as the average of the two-time correlation function over a period of the external driving, i.e.,

$$
C(\tau)=\frac{1}{T} \int_{0}^{T} d t\langle x(t+\tau) x(t)\rangle_{\infty} .
$$

The correlation function $C(\tau)$ can be written exactly as the sum of two contributions: a coherent part $C_{c o h}(\tau)$, which is periodic in $\tau$ with period $T$, and an incoherent part which decays to 0 for large $\tau$. The coherent part $C_{c o h}(\tau)$ is given by $[2,7]$

$$
C_{c o h}(\tau)=\frac{1}{T} \int_{0}^{T} d t\langle x(t+\tau)\rangle_{\infty}\langle x(t)\rangle_{\infty},
$$

where $\langle x(t)\rangle_{\infty}$ is the average value evaluated with the asymptotic form of the probability density $P_{\infty}(x, t)$.

It is possible to carry out a formal analysis of $C(\tau)$ and its coherent and incoherent components by making use of the spectral analysis of the Floquet operator associated with the Fokker-Planck dynamics. But an explicit evaluation of the correlation function is generally impossible; thus, one has to rely on numerical results obtained from integrating either the Langevin or the FPE, or by use of approximate analytical descriptions.

According to McNamara and Wiesenfeld [8], the output SNR is defined in terms of the Fourier transform of the coherent and incoherent parts of $C(\tau)$. As the correlation function is even in time and we evaluate its time dependence for $\tau \geqslant 0$, it is convenient to use its Fourier cosine transform, defined as

$$
\widetilde{C}(\omega)=\frac{2}{\pi} \int_{0}^{\infty} d \tau C(\tau) \cos (\omega \tau),
$$

$$
C(\tau)=\int_{0}^{\infty} d \omega \widetilde{C}(\omega) \cos (\omega \tau) .
$$

The value of the output SNR is then obtained from

$$
R_{\text {out }}=\frac{\lim _{\epsilon \rightarrow 0^{+}} \int_{\Omega-\epsilon}^{\Omega+\epsilon} d \omega \widetilde{C}(\omega)}{\widetilde{C}_{\text {incoh }}(\Omega)} .
$$

Note that this definition of the SNR differs by a factor 2, stemming from the same contribution at $\omega=-\Omega$, from the definitions used in earlier works [2,7]. The periodicity of the coherent part gives rise to $\delta$ peaks in the spectrum. Thus, the only contribution to the numerator in Eq. (10) stems from the coherent part of the correlation function. The evaluation of the SNR requires the knowledge of the Fourier components of $C_{c o h}(\tau)$ and $C_{\text {incoh }}(\tau)$ at the fundamental frequency of the driving force. Thus, rather than the entire Fourier spectrum, just two well defined numerical quadratures are needed. Namely,

$$
R_{o u t}=\frac{Q_{u}}{Q_{l}}
$$

where

$$
Q_{u}=\frac{2}{T} \int_{0}^{T} d \tau C_{c o h}(\tau) \cos (\Omega \tau)
$$

and

$$
Q_{l}=\frac{2}{\pi} \int_{o}^{\infty} d \tau C_{\text {incoh }}(\tau) \cos (\Omega \tau)
$$

The signal-to-noise ratio for an input signal $F(t)+\xi(t)$ is given by

$$
R_{\text {inp }}=\frac{\pi\left(f_{1}^{2}+g_{1}^{2}\right)}{4 D} .
$$

The so-called gain is defined as the ratio of the SNR of the output over the SNR of the input; namely,

$$
G=\frac{R_{\text {out }}}{R_{\text {inp }}} .
$$

\section{LINEAR RESPONSE THEORY BEYOND THE TWO-MODE APPROXIMATION}

The linear response theory provides a general procedure to describe the correlation function in an approximate way. The basic quantity of LRT is the system response function, $\chi(t)$. It is related to the equilibrium time correlation function of the system in the absence of external driving, $K(t)$, via the fluctuation-dissipation theorem (FDT) $[2,7,20,21]$, i.e.,

$$
\chi(t)= \begin{cases}0, & t \leqslant 0 \\ -\frac{1}{D} \dot{K}(t), & t>0 .\end{cases}
$$

The equilibrium time correlation function $K(t)$ is defined as 


$$
K(t)=\int_{-\infty}^{\infty} d x^{\prime} x^{\prime} P^{(e q)}\left(x^{\prime}\right) \int_{-\infty}^{\infty} d x x P_{1 \mid 1}^{(0)}\left(x, t \mid x^{\prime}\right),
$$

where $P^{(e q)}(x)$ is the equilibrium distribution of the nondriven system,

$$
P^{(e q)}(x)=N e^{-U(x) / D},
$$

and $P_{1 \mid 1}^{(0)}\left(x, t \mid x^{\prime}\right)$ is the conditional probability density to find, in the absence of driving, the variable near $x$ at time $t$, if it was initially at exactly $x^{\prime}$. Here we are assuming that the potential $U(x)$ is even in $x$, so that $\langle x\rangle_{e q}=0$.

Within LRT, the long time average value $\langle x(t)\rangle_{\infty}^{(L R T)}$ is given by

$$
\langle x(t)\rangle_{\infty}^{(L R T)}=\int_{0}^{\infty} d \tau \chi(\tau) F(t-\tau) .
$$

Insertion of the Fourier expansion Eq. (4) into Eq. (19) leads to

$$
\langle x(t)\rangle_{\infty}^{(L R T)}=\sum_{n=1}^{\infty}\left[M_{n}^{(L R T)} \cos (n \Omega t)+N_{n}^{(L R T)} \sin (n \Omega t)\right],
$$

where the coefficients $M_{n}^{(L R T)}$ and $N_{n}^{(L R T)}$ are given by

$$
M_{n}^{(L R T)}=f_{n} \chi_{n}^{(r)}-g_{n} \chi_{n}^{(i)}, \quad N_{n}^{(L R T)}=f_{n} \chi_{n}^{(i)}+g_{n} \chi_{n}^{(r)} .
$$

In these formulas, we have introduced the quantities $\chi_{n}^{(r)}$ and $\chi_{n}^{(i)}$ defined as

$$
\begin{aligned}
& \chi_{n}^{(r)}=\int_{0}^{\infty} d \tau \chi(\tau) \cos (n \Omega \tau), \\
& \chi_{n}^{(i)}=\int_{0}^{\infty} d \tau \chi(\tau) \sin (n \Omega \tau) .
\end{aligned}
$$

The use of the FDT in the above expressions allows us to write immediately

$$
\begin{gathered}
\chi_{n}^{(r)}=\frac{\left\langle x^{2}\right\rangle_{e q}-n \Omega \int_{0}^{\infty} d t K(t) \sin (n \Omega t)}{D}, \\
\chi_{n}^{(i)}=\frac{n \Omega}{D} \int_{0}^{\infty} d t K(t) \cos (n \Omega t) .
\end{gathered}
$$

It then follows from Eq. (8) that within LRT, the coherent part of the correlation function is given by

$$
C_{c o h}^{(L R T)}(\tau)=\frac{1}{2} \sum_{n=1}^{\infty}\left[\left(M_{n}^{(L R T)}\right)^{2}+\left(N_{n}^{(L R T)}\right)^{2}\right] \cos (n \Omega \tau) .
$$

As discussed in Refs. [2,7,20], LRT amounts to keeping the leading term in the perturbation treatment of the dynamics of the stochastic process $x(t)$ in powers of the driving ampli- tude. Then, within the spirit of perturbation theory, the leading term in the expansion of the incoherent part corresponds to the correlation function of the system in the absence of driving force, i.e., $C_{\text {incoh }}^{(L R T)}(\tau)=K(\tau)$.

Taking into account that $C_{c o h}^{(L R T)}(\tau)$ is periodic in $\tau$, it follows from Eqs. (9) and (26) that

$$
\begin{aligned}
\widetilde{C}_{c o h}^{(L R T)}(\omega)= & \frac{1}{2} \sum_{n=1}^{\infty}\left[\left(M_{n}^{(L R T)}\right)^{2}+\left(N_{n}^{(L R T)}\right)^{2}\right] \\
& \times[\delta(n \Omega-\omega)+\delta(n \Omega+\omega)] .
\end{aligned}
$$

Thus, it follows from the definition of the SNR, Eq. (10), that, within LRT, we have

$$
R^{(L R T)}=\frac{Q_{u}^{(L R T)}}{Q_{l}^{(L R T)}}
$$

where

$$
\begin{aligned}
Q_{u}^{(L R T)} & =\frac{1}{2}\left[\left(M_{1}^{(L R T)}\right)^{2}+\left(N_{1}^{(L R T)}\right)^{2}\right] \\
& =\frac{1}{2}\left(f_{1}^{2}+g_{1}^{2}\right)\left[\left(\chi_{1}^{(r)}\right)^{2}+\left(\chi_{1}^{(i)}\right)^{2}\right],
\end{aligned}
$$

and

$$
Q_{l}^{(L R T)}=\widetilde{K}(\Omega)=\frac{2 D \chi_{1}^{(i)}}{\pi \Omega}
$$

with $\widetilde{K}(\Omega)$ being the Fourier cosine transform of $K(t)$, defined according to Eq. (9). In arriving at Eqs. (28)-(30) we have also used Eqs. (21)-(23) and (25).

Taking into account Eqs. (14), (15) and (28)-(30), one readily finds that the gain within LRT is given by

$$
G^{(L R T)}=\frac{R_{\text {out }}^{(L R T)}}{R_{\text {inp }}}=\frac{\Omega\left[\left(\chi_{1}^{(r)}\right)^{2}+\left(\chi_{1}^{(i)}\right)^{2}\right]}{\chi_{1}^{(i)}} .
$$

This is a general expression for $G^{(L R T)}$ valid for any shape of the periodic driving signal.

The last expression will allow us to show that $G^{(L R T)}$ can, indeed, not exceed unity. Although this assertion has been discussed previously in Refs. $[9,10]$, we next will present a detailed and hopefully very clear proof for this prominent assertion.

As shown in the Appendix of Ref. [2], see also in Refs. $[11,12]$, the susceptibility $\chi(t)$ can be expressed as

$$
\chi(t)=-\sum_{p=1}^{\infty} e^{-\lambda_{p} t}\langle 0|x| p\rangle\left\langle p\left|\frac{\partial}{\partial x}\right| 0\right\rangle,
$$

where $|p\rangle=\psi_{p}(x),\langle p|=\psi_{p}^{\dagger}(x)$ and $\lambda_{p}$ are the eigenfunctions and eigenvalues of the FP operator $\hat{\mathcal{L}}_{0}$ associated to the undriven dynamics and its adjoint, $\hat{\mathcal{L}}_{0}^{\dagger}$, i.e.,

$$
\hat{\mathcal{L}}_{0} \psi_{p}(x)=-\lambda_{p} \psi_{p}(x), \quad \hat{\mathcal{L}}_{0}^{\dagger} \psi_{p}^{\dagger}(x)=-\lambda_{p} \psi_{p}^{\dagger}(x) .
$$


Using the above representation of the susceptibility in Eqs. (22) and (23) with $n=1$, we find

$$
\begin{aligned}
\chi_{1}^{(r)} & =-\sum_{p=1}^{\infty} \frac{\lambda_{p}}{\lambda_{p}^{2}+\Omega^{2}}\langle 0|x| p\rangle\left\langle p\left|\frac{\partial}{\partial x}\right| 0\right\rangle \\
& =\sum_{p=1}^{\infty} \frac{\lambda_{p}}{\lambda_{p}^{2}+\Omega^{2}}\left|\langle 0|x| p\rangle\left\langle p\left|\frac{\partial}{\partial x}\right| 0\right\rangle\right|, \\
\chi_{1}^{(i)} & =-\sum_{p=1}^{\infty} \frac{\Omega}{\lambda_{p}^{2}+\Omega^{2}}\langle 0|x| p\rangle\left\langle p\left|\frac{\partial}{\partial x}\right| 0\right\rangle \\
& =\sum_{p=1}^{\infty} \frac{\Omega}{\lambda_{p}^{2}+\Omega^{2}}\left|\langle 0|x| p\rangle\left\langle p\left|\frac{\partial}{\partial x}\right| 0\right\rangle\right| .
\end{aligned}
$$

Here, we have used the inequality

$$
\langle 0|x| p\rangle\left\langle p\left|\frac{\partial}{\partial x}\right| 0\right\rangle \leqslant 0,
$$

which can be proved as follows. Multiplying the first equation in Eq. (33) by $x$ and carrying out an integration by parts, one obtains

$$
\begin{aligned}
-\lambda_{p}\langle 0|x| p\rangle & =\int_{-\infty}^{\infty} d x x \hat{\mathcal{L}}_{0} \psi_{p}(x) \\
& =-\int_{-\infty}^{\infty} d x U^{\prime}(x) \psi_{0}(x) \psi_{p}^{\dagger}(x) \\
& =D \int_{-\infty}^{\infty} d x \psi_{p}^{\dagger}(x) \frac{\partial}{\partial x} \psi_{0}(x)=D\left\langle p\left|\frac{\partial}{\partial x}\right| 0\right\rangle,
\end{aligned}
$$

where we have taken into account that $\psi_{0}(x)=P^{(e q)}(x)$ and $\psi_{p}(x)=\psi_{0}(x) \psi_{p}^{\dagger}(x)$, so that $\psi_{0}^{\dagger}(x)=1$. Therefore, $\langle 0|x| p\rangle$ $\times\langle p|\partial / \partial x| 0\rangle=-\lambda_{p}(\langle 0|x| p\rangle)^{2} / D \leqslant 0$. Using in Eqs. (34) and (35) the Cauchy-Schwarz inequality, we find

$$
\begin{aligned}
\left(\chi_{1}^{(r)}\right)^{2}= & {\left[\sum_{p=1}^{\infty} \frac{\lambda_{p}\left|\langle 0|x| p\rangle\left\langle p\left|\frac{\partial}{\partial x}\right| 0\right\rangle\right|^{1 / 2}}{\lambda_{p}^{2}+\Omega^{2}}\right.} \\
& \left.\times\left|\langle 0|x| p\rangle\left\langle p\left|\frac{\partial}{\partial x}\right| 0\right\rangle\right|^{1 / 2}\right]^{2} \\
\leqslant & \sum_{p=1}^{\infty} \frac{\lambda_{p}^{2}\left|\langle 0|x| p\rangle\left\langle p\left|\frac{\partial}{\partial x}\right| 0\right\rangle\right|}{\left(\lambda_{p}^{2}+\Omega^{2}\right)^{2}} \sum_{q=1}^{\infty}\left|\langle 0|x| q\rangle\left\langle q\left|\frac{\partial}{\partial x}\right| 0\right\rangle\right|,
\end{aligned}
$$

$$
\begin{aligned}
\left(\chi_{1}^{(i)}\right)^{2}= & {\left[\sum_{p=1}^{\infty} \frac{\Omega\left|\langle 0|x| p\rangle\left\langle p\left|\frac{\partial}{\partial x}\right| 0\right\rangle\right|^{1 / 2}}{\lambda_{p}^{2}+\Omega^{2}}\right.} \\
& \left.\times\left|\langle 0|x| p\rangle\left\langle p\left|\frac{\partial}{\partial x}\right| 0\right\rangle\right|^{1 / 2}\right]^{2} \\
\leqslant & \sum_{p=1}^{\infty} \frac{\Omega^{2}\left|\langle 0|x| p\rangle\left\langle p\left|\frac{\partial}{\partial x}\right| 0\right\rangle\right|}{\left(\lambda_{p}^{2}+\Omega^{2}\right)^{2}} \sum_{q=1}^{\infty}\left|\langle 0|x| q\rangle\left\langle q\left|\frac{\partial}{\partial x}\right| 0\right\rangle\right| .
\end{aligned}
$$

Taking into account that $\langle 0|x| 0\rangle=0$, the completeness relation yields $\sum_{q=1}^{\infty}|\langle 0|x| q\rangle\langle q|\partial / \partial x| 0\rangle|$ $=-\Sigma_{q=0}^{\infty}\langle 0|x| q\rangle\langle q|\partial / \partial x| 0\rangle=-\langle 0|x \partial / \partial x| 0\rangle=1$. Thus, by adding Eq. (38) to Eq. (39), one obtains

$$
\left(\chi_{1}^{(r)}\right)^{2}+\left(\chi_{1}^{(i)}\right)^{2} \leqslant \sum_{p=1}^{\infty} \frac{\left|\langle 0|x| p\rangle\left\langle p\left|\frac{\partial}{\partial x}\right| 0\right\rangle\right|}{\lambda_{p}^{2}+\Omega^{2}}=\frac{\chi_{1}^{(i)}}{\Omega} .
$$

Finally, inserting Eq. (40) into (31), we obtain the seminal inequality that $G^{(L R T)} \leqslant 1$.

Put differently, the gain of a nonlinear system operating in a regime where LRT provides a valid description cannot reach values greater than 1 . This result is valid for any periodic external driving. Notice that this finding does not preclude the possibility of obtaining values for the SNR gain larger than unity when the conditions are such that the use of LRT is not sensible.

\section{NUMERICAL RESULTS}

In this section, we will carry out the numerical evaluation of the different magnitudes defined above. Our goal is to compare the predictions of LRT with the results obtained from the numerical solution of the Langevin equation, Eq. (1). We will consider the dynamics in the bistable potential $U(x)=-x^{2} / 2+x^{4} / 4$ driven by time-periodic forces.

The evaluation of the different magnitudes using LRT requires the knowledge of $K(t)$ [cf. Eqs. (21), (26), and (28)(31)]. For nonlinear problems, explicit expressions for $K(t)$ are unknown, but useful approximations have been presented in the literature. For the bistable potential, $U(x)=-x^{2} / 2$ $+x^{4} / 4$, Jung and Hänggi [21] have used the two-mode approximation. It is based on the existence of a large difference in the time scales associated to interwell and intrawell motions, and it is expected to be valid for small values of the noise strength $D$. With this model, one finds

$$
K(\tau)=g_{1} e^{-\lambda_{1} \tau}+g_{2} e^{-\alpha \tau},
$$

where [2]

$$
\lambda_{1} \approx \frac{\sqrt{2}}{\pi}\left(1-\frac{3}{2} D\right) \exp [-1 /(4 D)],
$$


and $\alpha=2$. The weights $g_{1}$ and $g_{2}$ can be obtained from the moments of the equilibrium distribution in the absence of driving using the expressions

$$
\begin{gathered}
g_{2}=\frac{\lambda_{1}\left\langle x^{2}\right\rangle_{e q}}{\lambda_{1}-\alpha}+\frac{\left\langle x^{2}\right\rangle_{e q}-\left\langle x^{4}\right\rangle_{e q}}{\lambda_{1}-\alpha}, \\
g_{1}=\left\langle x^{2}\right\rangle_{e q}-g_{2} .
\end{gathered}
$$

To leading order in $D$, we can replace $\lambda_{1}$ by $\lambda_{K}$ $=\sqrt{2} / \pi \exp [-1 /(4 D)], g_{1} \approx 1$ and $g_{2} \approx D / \alpha$. This is the limit considered in Ref. [26]. In the results reported below, we have also considered values of $D$ so large that the twomode approximation becomes inadequate. Therefore, the correlation function in the absence of driving has been evaluated numerically from the FPE in the absence of driving following the procedure discussed in Ref. [24].

The numerical evaluation of the correlation function $C(\tau)$ and its coherent and incoherent parts proceeds as follows. Stochastic trajectories $x^{(j)}(t)$ are generated by numerically integrating the Langevin equation for every realization $j$ of the white noise $\xi(t)$, starting from a given initial condition $x_{0}$. The numerical solution is based on the algorithm developed by Greenside and Helfand [27,28]. The essence of the algorithm is briefly sketched in the Appendix. After allowing for a relaxation transient stage, we start recording the time evolution of each random trajectory for many different trajectories. Then, we construct the two-time ( $t$ and $\tau$ ) correlation function, i.e.,

$$
\langle x(t+\tau) x(t)\rangle_{\infty}=\frac{1}{N} \sum_{j=1}^{N} x^{(j)}(t+\tau) x^{(j)}(t),
$$

as well as the product of the averages

$$
\langle x(t+\tau)\rangle_{\infty}\langle x(t)\rangle_{\infty}=\left(\frac{1}{N} \sum_{j=1}^{N} x^{(j)}(t+\tau)\right)\left(\frac{1}{N} \sum_{j=1}^{N} x^{(j)}(t)\right),
$$

where $N$ is the number of stochastic trajectories considered. The correlation function $C(\tau)$ and its coherent part $C_{c o h}(\tau)$ are then obtained using their definitions in Eqs. (7) and (8), performing the cycle average over one period of $t$. The difference between the values of $C(\tau)$ and $C_{c o h}(\tau)$ allows us to obtain the values for $C_{\text {incoh }}(\tau)$. It is then straightforward to evaluate the Fourier component of $C_{c o h}(\tau)$ and the Fourier transform of $C_{\text {incoh }}(\tau)$ at the driving frequency by numerical quadrature. With that information, the numerator and the denominator for the output SNR [cf. Eqs. (11)-(13)], as well as the gain [cf. Eq. (15)], are obtained.

We shall analyze two different types of periodic driving forces. First, let us consider the well known situation with a monochromatic, single-frequency force, $A \cos (\Omega t)$, with amplitude strength $A$ and angular frequency $\Omega$ [2]. In this case, the formulas in Sec. III simplify considerably because $f_{1}$ $=A$, while all the other Fourier components of the driving force vanish. The second case corresponds to a periodic force with period $T$, with a sequence of pulses of length $t_{c}<T / 2$, namely,

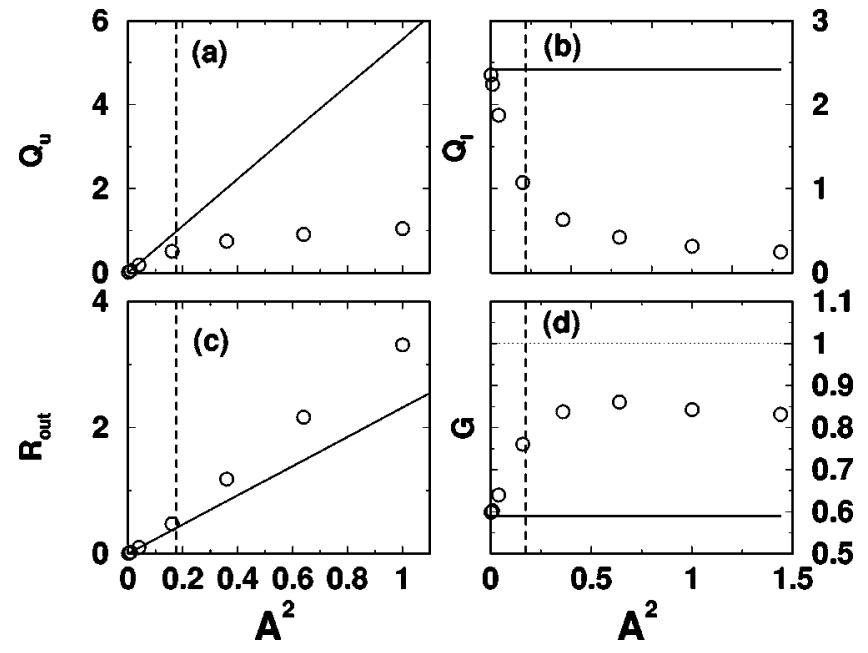

FIG. 1. The dependence of several SR quantifiers vs the square of the driving amplitude, $A^{2}$, given by LRT (solid line) and by the numerical solution of the Langevin equation (circles). In panels (a) and (b), we plot, respectively, the numerator and denominator appearing in the definition of output SNR, cf. Eqs. (11) and (28). The behaviors of the output SNR and the gain are depicted, respectively, in panels (c) and (d). The driving force is monochromatic with frequency $\Omega=0.1$ and the white noise strength is kept constant at the value $D=0.2$. In all panels, the vertical dashed line indicates the square of the value of the dynamical threshold amplitude $A_{t h}$ at the angular driving frequency $\Omega$. In panel (d), a dotted horizontal line is drawn at the gain value of 1 as a guide to the eye.

$$
F(t)= \begin{cases}A, & 0 \leqslant t<t_{c} \\ -A, & \frac{T}{2} \leqslant t<\frac{T}{2}+t_{c} \\ 0, & \text { otherwise. }\end{cases}
$$

In this case, we have

$$
f_{1}=\frac{2 A}{\pi} \sin \left(\Omega t_{c}\right), \quad g_{1}=\frac{2 A}{\pi}\left[1-\cos \left(\Omega t_{c}\right)\right]
$$

where $\Omega=2 \pi / T$ is the fundamental frequency. This force is characterized by its amplitude, its period, and its duty cycle, which is defined as $2 t_{c} / T$. Recently, Gingl et al. $[18,19]$ have carried out analog simulations of systems that are subjected to wideband Gaussian noise and driving forces of this second type. They report values for the gain that greatly exceeds unity, for driving amplitudes below its threshold value. If this is the case, then strong deviations from the LRT should be observed as well.

\section{A. Monochromatic driving}

In Fig. 1, we depict the results obtained for a monochromatic driving force with angular frequency $\Omega=0.1$, noise strength $D=0.2$, and several values of the amplitude. In the deterministic dynamics $(D=0)$, an external periodic force with the indicated frequency induces sustained oscillations between the minima of the potential for $A \geqslant A_{t h} \simeq 0.419$. Note that this nonadiabatic frequency raises the threshold 
value for superthreshold driving beyond its adiabatic lower limit of $A_{t h}^{(a d)}=\sqrt{4 / 27} \simeq 0.3849$. Thus, we will take this value as the amplitude threshold value at the frequency $\Omega=0.1$. In panel (a), we plot the numerators, $Q_{u}$ and $Q_{u}^{(L R T)}$, of the output SNR given by Eqs. (12) and (29) vs $A^{2}$. The solid straight line represents the LRT result, while the circles correspond to the numerical results. The graph reveals that for amplitude strengths $A<0.1$ the predictions of LRT match well the numerical results, as can be expected. When the amplitude increases, the deviations of LRT from the precise numerical results are large. LRT predicts a much larger amplification of the output amplitude than the one obtained numerically. In panel (b), we plot the denominators, $Q_{l}$ and $Q_{l}^{(L R T)}$, of the output SNR given by Eqs. (13) (circles) and (30) (solid line) vs $A^{2}$. In LRT, the denominator is independent of $A$. Once again, the predictions of LRT match the numerical results for $A<0.1$. For larger values of $A$, the influence of the driving amplitude on the relaxation of $C_{\text {incoh }}(\tau)$ is very strong and the numerical results for the denominator are much smaller than the ones obtained within LRT. It is then clear that LRT will yield a valid description of the signal-to-noise ratio for small driving amplitudes only as depicted in panel (c). We notice that the values of $R_{\text {out }}$ provided by the numerics are larger than those of $R_{\text {out }}^{(L R T)}$. This is so although linear response theory predicts larger spectral amplifications, see in Ref. [7], of the average output than what really occurs. The modifications in the behavior of the incoherent part of the correlation function with respect to its behavior in the absence of driving are more than enough to compensate for the behavior of the numerators. In panel (d), we plot the gain vs $A^{2}$. There exists an optimum value for the driver amplitude $(A \sim 0.8)$ at which the gain becomes maximized. Nonetheless, the gain is always smaller than unity. LRT requires that $G^{(L R T)} \leqslant 1$. These strong deviations of the predictions of LRT about the behavior of the two components of the correlation function with respect to the numerical results tell us that LRT cannot be invoked to explain the fact that the gain is smaller than 1 for the range of parameter values considered in this figure; that is, a gain below 1 occurs here within the nonlinear regime.

In Figs. 2 and 3 we analyze the same quantities as in Fig. 1 , but now for larger noise values, $D=0.6$ and $D=1.0$, respectively. The most important difference with respect to the plots in Fig. 1 is that for these larger values of the noise, the gain can exceed unity for values of the amplitude well above its threshold value. This superthreshold feature has been corroborated already in Ref. [17]; a gain above 1 seemingly does not occur for monochromatic subthreshold driving.

\section{B. Pulsed, multichromatic periodic driving}

Next, we proceed to consider the case of pulsed driving forces. In Figs. 4 and 5, we compare the dependence of the output on the driving amplitude as given by the LRT approximation with the numerical precise results. The system is forced by a multifrequency driver with a period $T=2 \pi / 0.1$ $\simeq 63$ and a duty cycle of $10 \%$. As in the case of a singlefrequency driving, the values of the different quantities obtained from the numerics deviate significantly from the pre-

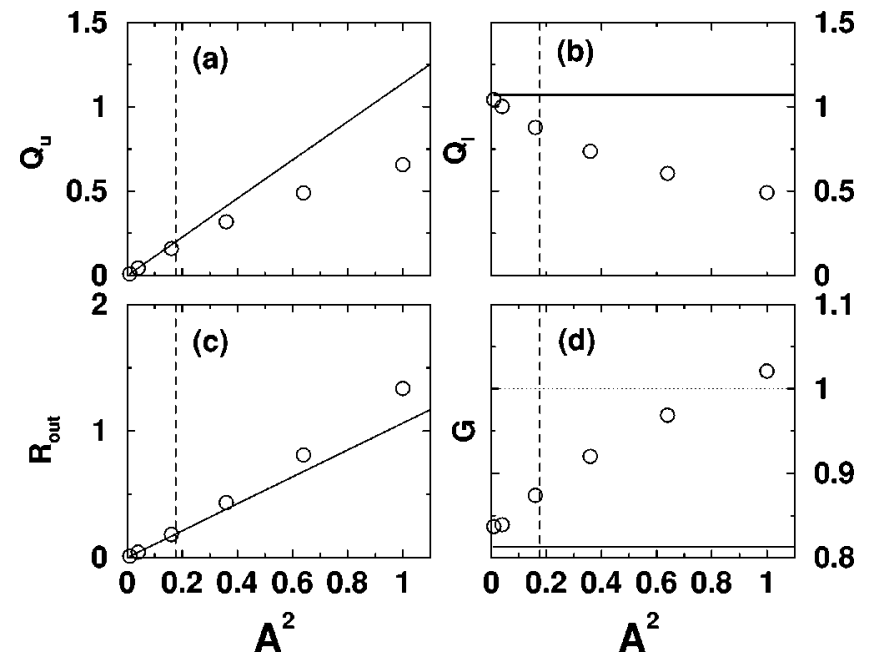

FIG. 2. The same as in Fig. 1 but now for $\Omega=0.1$ and $D=0.6$.

dictions of LRT as the amplitude of the driver is increased. Nevertheless, perhaps the most relevant difference with respect to the monochromatic case is that we again do not find gains larger than 1 in the range of parameter values considered in these figures.

\section{The case of strong nonlinearity}

A particularly interesting situation arises in the analog studies of pulsed driving forces with a very small fundamental frequency: in Refs. $[18,19]$ Gingl et al. report gains that significantly exceed the value 1 for a subthreshold, multifrequency driving force of very large period $T=2 \pi / 0.0024$ $\simeq 2618$ and a small duty cycle of $10 \%$. This large gain is accompanied by a nonmonotonic behavior of the SNR with the noise strength $D$. Therefore, this situation must correspond to a very sensible discrepancy of the actual behavior with respect to the LRT predictions. We have carried out detailed and careful numerics of the Langevin equation in this extreme regime for such a driving force with a sub-

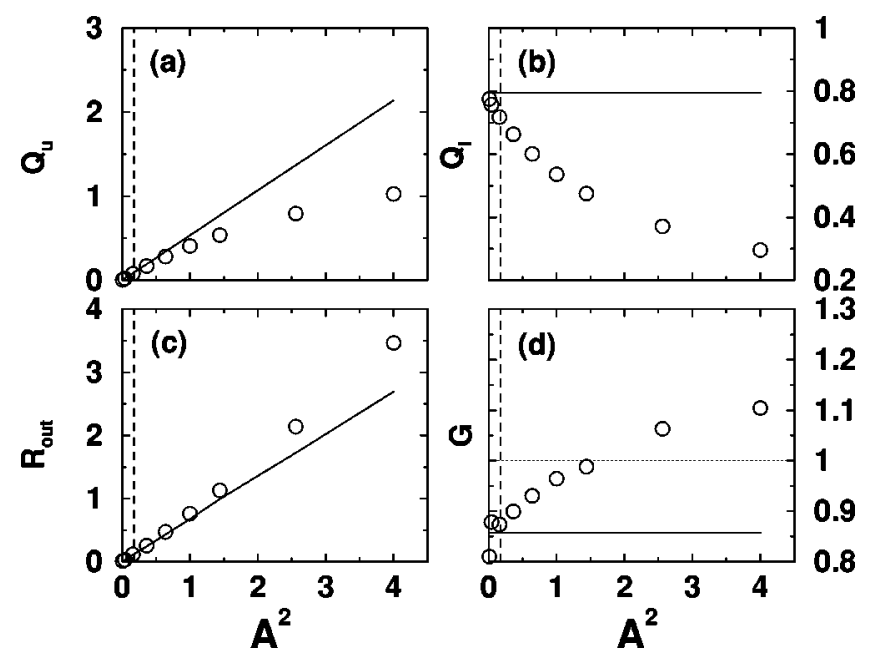

FIG. 3. The same as in Fig. 1 but now for $\Omega=0.1$ and $D=1.0$. 

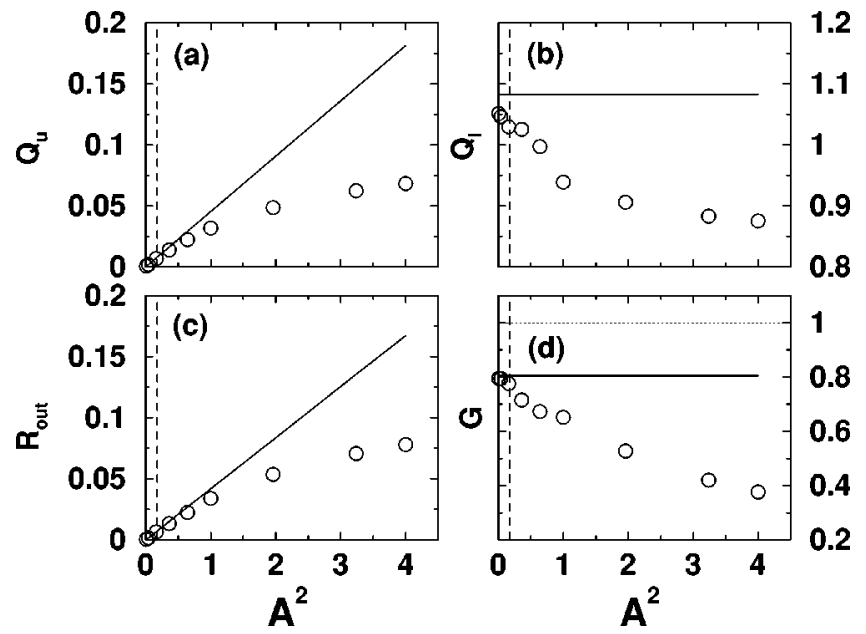

FIG. 4. The same as in Fig. 1, for the case of a pulsed, driving force with period $T \simeq 63$, duty cycle $2 t_{c} / T=0.1$, cf. Eq. (47), and a noise strength $D=0.6$.

threshold amplitude $A=0.35$ and a noise strength $D=0.02$. With the parameters considered, the problem becomes computationally very demanding indeed: this is so because of the very large period of the driving force. Moreover, in order to obtain reliable numerical results for the incoherent part of the correlation function a large number of stochastic trajectories needs to be generated. Our findings are summarized in Table I.

To obtain a reliable convergence of the corresponding SR quantifiers, at least up to 50000 random trajectories need to be considered. A smaller sampling size can induce severe errors, see in Table I. The main result is a numerically evaluated gain of 8.62; in clear contrast, the result predicted by LRT is the very small value of 0.018 ; that is, LRT strikingly fails, cf. in Table I for the corresponding values of SNR and its constituents. The SNR value of the analog simulation in Refs. $[18,19]$ carried out with a pulsed input signal with the same characteristics as the one considered here, and wide-

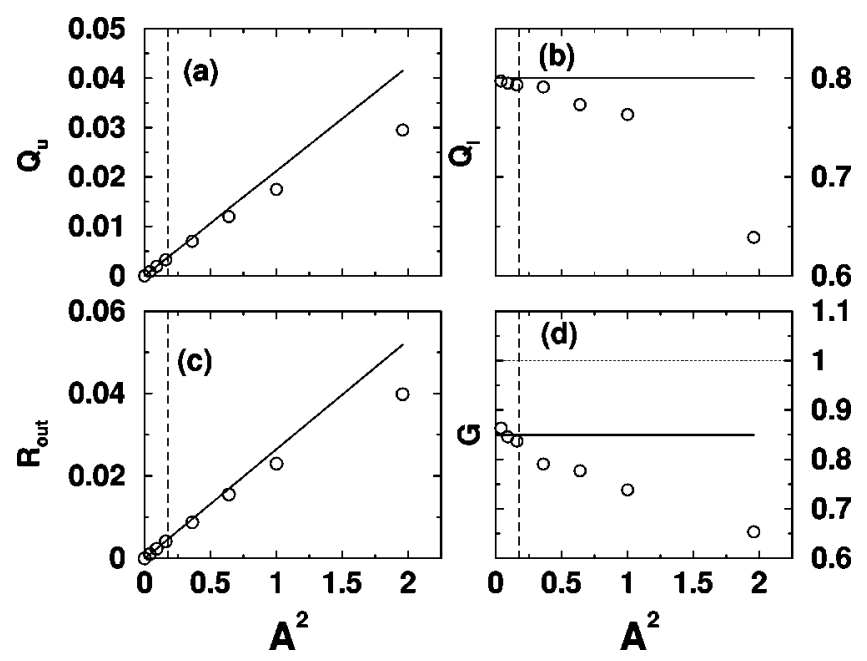

FIG. 5. The same as in Fig. 1 for a pulsed driving force with period $T \simeq 63$, duty cycle $2 t_{c} / T=0.1$, cf. Eq. (47), and a noise strength $D=1.0$.
TABLE I. Numerically obtained values of several quantities for different numbers of noise realizations and their LRT results.

\begin{tabular}{lccccc}
\hline \hline \multirow{2}{*}{ Numerics } & Trajectories & $Q_{u}$ & $Q_{l}$ & $R_{\text {out }}$ & $G$ \\
& 1000 & 0.78 & 0.33 & 2.32 & 12.16 \\
& 5000 & 0.78 & 0.35 & 2.26 & 11.84 \\
& 10000 & 0.78 & 0.47 & 1.67 & 8.77 \\
\multirow{4}{*}{ LRT } & 50000 & 0.78 & 0.48 & 1.65 & 8.62 \\
& & 0.00061 & 0.177 & 0.0034 & 0.018 \\
\hline \hline
\end{tabular}

band Gaussian noise with a related strength roughly similar to ours, yields an experimentally determined gain of ca. 19, cf. Fig. 4 in Ref. [19]. This value is again significantly larger than 1 and compares favorably with our results in Table I. Note, however, that the sampling size of ca. 1000 realizations used in Refs. $[18,19]$ has been chosen substantially smaller than the number of realizations needed to achieve good numerical convergence, cf. Table I; this in turn may explain the overshoot of the experimentally determined gain value.

\section{CONCLUSIONS}

Let us summarize the main results of this work.

(i) First, we have provided an analytical proof based on LRT beyond the commonly employed two-mode approximation that the gain of a noisy, periodically driven nonlinear system which operates within the regime of validity of LRT cannot exceed unity. This result holds for arbitrary noise strength $D$ and is independent of the shape of the input signal.

(ii) We have implemented a very efficient algorithm due to Greenside and Helfand $[27,28]$ to numerically integrate the Langevin equation. From the numerical solution, we have evaluated the time evolution of the correlation function and its coherent and incoherent components.

(iii) We have also put forward a procedure, alternative to the usual one, to calculate the SNR. The numerator and denominator of the SNR are calculated by use of only two numerical quadratures.

(iv) A detailed comparison between the predictions of LRT and the numerical results have been carried out. We have assessed regions of parameter values where LRT gives an erroneous description, yet the gain, nevertheless, is less than unity. On the other hand, there exist regions in parameter space where the gain indeed exceeds 1 if driven with a superthreshold amplitude strength; this finding is in agreement with prior results in Ref. [17]. These regions are again characterized by substantial deviations from LRT.

Moreover, as previously established by use of analog simulations by Gingl et al. $[18,19]$ we also find the surprising result, valid for dynamical systems, that SNR gains larger than unity can indeed occur for subthreshold multichromatic input signals: For this feature to occur one seemingly needs, however, weak noise and a slow periodic driving signal with a very small duty cycle. In this context, the necessity of a sufficiently large number of sampling trajectories in order to obtain reliable, convergent results has also been stressed. It is 
in this very regime of small frequency driving and weak noise where the LRT description indeed fails notably $[11,12]$.

\section{ACKNOWLEDGMENTS}

We acknowledge the support of the Dirección General de Enseñanza Superior of Spain (Grant No. BFM2002-03822), the Junta de Andalucía, the DAAD program "Acciones Integradas" (P.H., M.M.), and the Sonderforschungsbereich 486 of the Deutsche Forschungsgemeinschaft, Project No. A10.

\section{APPENDIX: THE METHOD OF GREENSIDE AND HELFAND}

The procedure proposed by Greenside and Helfand for numerically integrating stochastic differential equations has been discussed in detail by them in Refs. [27,28]. For the sake of completeness, we will briefly sketch in this appendix the main reasoning of their procedure. By analogy with deterministic Runge-Kutta algorithms, Greenside and Helfand developed schemes to estimate the value of the stochastic variable at time $t+h$ if its value at time $t$ is known. This is achieved by evaluating the right hand side of the Langevin equation at selected points within each interval of length $h$, so that all moments of $x(t+h)-x(t)$ are correct to order $h^{k}$.

As our Langevin equation contains an explicit time dependent driving force, it is convenient to rewrite it as a twodimensional problem with variables $\left(y_{1}, y_{2}\right)=\vec{y}$, where $y_{1}$ $=x$ and $y_{2}=t$. The Langevin equation, Eq. (1), is then written in vector form as

$$
\frac{d \vec{y}}{d t}=\vec{G}(\vec{y})+\vec{\Xi}(t)
$$

where $\vec{G}=\left(G_{1}, G_{2}\right)=\left(-U^{\prime}(x)+F(t), 1\right) \quad$ and $\quad \vec{\Xi}(t)$ $=(\xi(t), 0)$.

The formal solution of Eq. (A1) yields

$$
y_{\kappa}(h)=y_{\kappa}(0)+\int_{0}^{h} d s G_{\kappa}(\vec{y}(s)) s+w_{\kappa}^{(0)}(h) \quad(\kappa=1,2)
$$

with

$$
w_{\kappa}^{(0)}(h)=\int_{0}^{h} d s \Xi_{\kappa}(s) .
$$

The right hand side of Eq. (A2) can be expanded as

$$
\begin{aligned}
y_{\kappa}(h)= & y_{\kappa}(0)+h G_{\kappa}(\vec{y}(0))+\frac{1}{2} h^{2} \sum_{\mu} \frac{\partial G_{\kappa}(\vec{y}(0))}{\partial y_{\mu}} G_{\mu}(\vec{y}(0)) \\
& +\cdots+S_{\kappa}(h) .
\end{aligned}
$$

The last term $S_{K}(h)$ represents the stochastic part. It is a series in $h^{1 / 2}$ with the order of the terms determined in probability.
TABLE II. Parameter values given by Greenside and Helfand [28] for their $3 O_{O}{ }_{S} 2_{G}$ algorithm.

\begin{tabular}{cccc}
\hline \hline$A_{1}$ & 0.0 & $A_{2}$ & 0.644468 \\
$A_{3}$ & 0.194450 & $A_{4}$ & 0.161082 \\
$\beta_{21}$ & 0.516719 & $\beta_{31}$ & -0.397300 \\
$\beta_{32}$ & 0.427690 & $\beta_{41}$ & -1.587731 \\
$\beta_{42}$ & 1.417263 & $\beta_{43}$ & 1.170469 \\
$\lambda_{01}$ & 1.0 & $\lambda_{02}$ & 0.0 \\
$\lambda_{11}$ & 0.0 & $\lambda_{12}$ & 0.271608 \\
$\lambda_{21}$ & 0.516719 & $\lambda_{22}$ & 0.499720 \\
$\lambda_{31}$ & 0.030390 & $\lambda_{32}$ & -0.171658 \\
$\lambda_{41}$ & 1.0 & $\lambda_{42}$ & 0.0 \\
\hline \hline
\end{tabular}

By analogy with the Runge-Kutta procedures for deterministic differential equations, Greenside and Helfand propose an $l$-stage algorithm to write the solution of Eq. (A1) as

$$
y_{\kappa}(h)=y_{\kappa}(0)+h\left(A_{1} g_{1 \kappa}+\cdots+A_{l} g_{l \kappa}\right)+h^{1 / 2} \Xi_{\kappa}^{1 / 2} Y_{0 \kappa},
$$

with

$$
\begin{aligned}
g_{1 \kappa}= & G_{\kappa}\left(\left\{y_{\mu}(0)+h^{1 / 2} \Xi_{\mu}^{1 / 2} Y_{1 \mu}\right\}\right), \\
g_{2 \kappa}= & G_{\kappa}\left(\left\{y_{\mu}(0)+h \beta_{21} g_{1 \mu}+h^{1 / 2} \Xi_{\mu}^{1 / 2} Y_{2 \mu}\right\}\right), \\
\vdots & \\
g_{l \kappa}= & G_{\kappa}\left(\left\{y_{\mu}(0)+h \beta_{l 1} g_{1 \mu}+\cdots+h \beta_{l, l-1} g_{l-1, \mu}\right.\right. \\
& \left.\left.+h^{1 / 2} \Xi_{\mu}^{1 / 2} Y_{l \mu}\right\}\right) .
\end{aligned}
$$

Here, $\left(\left\{y_{\mu}\right\}\right)$ is the set $(x, t)$. The $Y_{l \mu}$ are Gaussian stochastic variables with zero average, which are numerically generated by writing

$$
Y_{i \kappa}=\sum_{j=1}^{m} \lambda_{i j} Z_{j \kappa}
$$

where $Z_{j \kappa}$ are $m$ independent Gaussian random variables of zero average and unit variance. The parameters $A_{i}, \beta_{i j}$, and $\lambda_{i j}$ appearing in Eqs. (A5)-(A7) are independent of the component index $\kappa$. They are obtained by expanding Eq. (A5) to the desired order $h^{k}$. This expansion gives rise to a deterministic and a stochastic part, $\widetilde{S}_{\kappa}$. Equating the coefficients of this expansion with those of the deterministic part in Eq. (A4) leads to a set of equations for the parameters $A_{i}, \beta_{i j}$, and $\lambda_{i j}$. Further equations are obtained by equating the moments of $\left\langle\widetilde{S}_{\kappa}^{n}\right\rangle$ with those of the stochastic part in the expansion in Eq. (A4) $\left\langle S_{\kappa}^{n}\right\rangle$.

A procedure correct to order $h^{k}$ in the step size $h$, involving $l$ stages and $m$ Gaussian independent variables, is termed a $k_{O} l_{S} m_{G}$ algorithm. In this paper, we have integrated the Langevin equation using a $3{ }_{O} 4{ }_{S} 2_{G}$ algorithm with the values for $A_{i}, \beta_{i j}$, and $\lambda_{i j}$ given in Table II taken from Ref. [28]. With this choice of parameters, the deterministic part is of order $h^{4}$, as in the fourth-order Runge-Kutta procedure for ordinary differential equations. 
[1] A.R. Bulsara and L. Gammaitoni, Phys. Today 49(3), 39 (1996).

[2] L. Gammaitoni, P. Hänggi, P. Jung, and F. Marchesoni, Rev. Mod. Phys. 70, 223 (1998).

[3] K. Wiesenfeld and F. Jaramillo, Chaos 8, 539 (1998).

[4] V.S. Anishchenko, A.B. Neiman, F. Moss, and L. SchimanskyGeier, Usp. Fiz. Nauk 169, 7 (1999) [Phys. Usp. 42, 7 (1999)].

[5] P. Hänggi, ChemPhysChem 3, 285 (2002).

[6] P. Jung and P. Hänggi, Europhys. Lett. 8, 505 (1989).

[7] P. Jung and P. Hänggi, Phys. Rev. A 44, 8032 (1991).

[8] B. McNamara and K. Wiesenfeld, Phys. Rev. A 39, 4854 (1989).

[9] M.I. Dykman, D.G. Luchinsky, R. Mannella, P.V.E. McClintock, N.D. Stein, and N.G. Stocks, Nuovo Cimento Soc. Ital. Fis., D 17D, 660 (1995).

[10] M. DeWeese and W. Bialek, Nuovo Cimento Soc. Ital. Fis., D 17D, 733 (1995).

[11] J. Casado-Pascual, J. Gómez-Ordóñez, M. Morillo, and P. Hänggi, Europhys. Lett. 58, 342 (2002).

[12] J. Casado-Pascual, J. Gómez-Ordóñez, M. Morillo, and P. Hänggi, Fluct. Noise Lett. 2, L127 (2002).

[13] K. Loerincz, Z. Gingl, and L.B. Kiss, Phys. Lett. A 224, 63 (1996).

[14] F. Chapeau-Blondeau and X. Godivier, Phys. Rev. E 55, 1478 (1997).
[15] Z. Gingl, R. Vatjai, and L.B. Kiss, Chaos, Solitons Fractals 11, 1929 (2000).

[16] F. Liu, Y. Yu, and W. Wang, Phys. Rev. E 63, 051912 (2001).

[17] P. Hänggi, M. Inchiosa, D. Fogliatti, and A.R. Bulsara, Phys. Rev. E 62, 6155 (2000).

[18] Z. Gingl, R. Vajtai, and P. Makra, in ICNF 2001, edited by G. Bosman (World Scientific, Singapore, 2002), pp. 545-548.

[19] Z. Gingl, P. Makra, and R. Vajtai, Fluct. Noise Lett. 1, L181 (2001).

[20] P. Hänggi and H. Thomas, Phys. Rep. 88, 207 (1982), see Sec. 5, pp. 275-287 therein.

[21] P. Jung and P. Hänggi, Z. Phys. B: Condens. Matter 90, 255 (1993).

[22] M. Morillo and J. Gómez-Ordóñez, Phys. Rev. E 51, 999 (1995).

[23] M.D. Feit, J.A. Fleck, Jr., and A. Steiger, J. Comput. Phys. 47, 412 (1982).

[24] M. Morillo and J. Gómez-Ordóñez, Phys. Rev. A 46, 6738 (1992).

[25] W.H. Press, S.A. Teukolsky, W.T. Vetterling, and B.P. Flannery, Numerical Recipes, 2nd ed. (Cambridge University Press, Cambridge, UK, 1992), Chap. 13.

[26] G. Hu, H. Haken, and C.Z. Ning, Phys. Lett. A 172, 21 (1992).

[27] E. Helfand, Bell Syst. Tech. J. 58, 2289 (1979).

[28] H.S. Greenside and E. Helfand, Bell Syst. Tech. J. 60, 1927 (1981). 